\title{
Supporting Mood Awareness in Collaborative Settings
}

\author{
Simone Mora \\ Department of Computer and Information Science \\ Norwegian University of Science and Technology \\ Trondheim, Norway \\ simonem@idi.ntnu.no
}

\author{
Verónica Rivera-Pelayo, Lars Müller \\ FZI Forschungszentrum Informatik \\ Karlsruhe, Germany \\ \{rivera,1mueller\}@fzi.de
}

\begin{abstract}
Affective aspects during collaboration can be exploited as triggers for reflection, yet current tools usually ignore these aspects. In this paper, we present a set of design choices to inform the design of systems towards enabling mood awareness in collaborative work settings like meetings or conferences. Design choices have served to outline and implement a collection of prototypes, including two input interfaces and three visualizations, which have been evaluated during an important project meeting over three days. Our results show that (a) the user acceptance of capturing mood is high (b) the aggregated mood values are related to the work process and (c) aggregated moods can influence the individual by creating awareness of others. Further discussion on the impact of the different design choices shows promising venues to improve mood awareness support.
\end{abstract}

Mood Awareness; Collaborative Tools; User centered design; User Interfaces

\section{INTRODUCTION}

Despite feelings and emotions constitute an important part of any experience [1], research in Computer Supported Collaborative Work (CSCW) often ignores them. The line of research in awareness aims to support collaborative work by providing awareness of elements of context considered relevant to get an ongoing activity done, e.g. location or participants status [2][3][4]; but giving awareness cues of how people feel during that activity is often not considered.

Being aware of how participants feel during an activity is important to enable reflection processes that might result in better work practices and behavior changing. According with the model of Boud et al (1985) [5], people re-evaluate a past experience by considering its various aspects (including affective ones) towards a learning outcome (Figure 1).

A key aspect in making a reflective process to happen is the presence of triggers. Mood awareness brings important triggers for reflection; e.g. a sudden drop of mood during a work shift might induce in reflecting on what activity/event has provoked it, why, and how to get things done better in the future.

This work has been co-funded by the European Commission within the 7th Framework Programme in the MIRROR project (http://www.mirrorproject.eu).

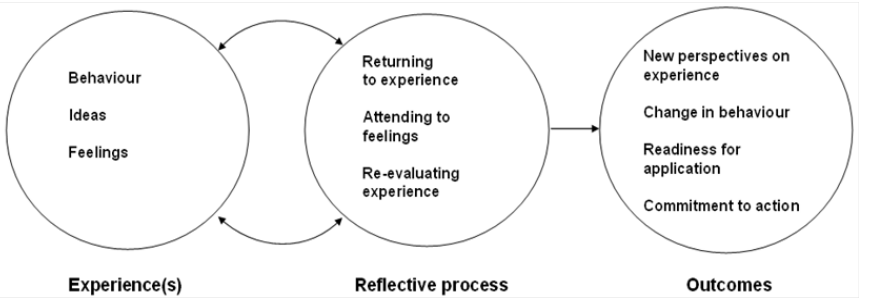

Figure 1: The process of reflective learning (Boud et al. 1985)

During any co-located collaborative activity, we use visual cues to relate our own mood to our peers' in order to find an endorsement of our feelings and adapt our behavior; despite social conventions often lead us to not exhibit our feelings. Sharing feelings and being aware of our fellows' moods may trigger reflection by allowing comparing a personal feeling with others; discrepancies in the moods of the groups may for example lead the individual to re-evaluate an experience from other points of view. For example a keynote speaker can check his own mood against the audience's mood in order to evaluate the interest raised by her talk.

Recent advances in mobile and ubiquitous technology offer the possibility to design tools that promote mood awareness in collaborative work settings, thus enriching the awareness modalities that are currently available to cooperating actors.

Our main focus is in exploring the design space of such applications. We identified a set of design choices to be taken into account in building systems that deal with emotions. We implemented several prototypes, exploiting mobile and calm technologies [6], towards the goal of providing a seamless integration of input interfaces and visualization tools to achieve continuous mood awareness. We tested our applications against a scenario and we discuss the feedbacks collected from real users in order to analyze design trade-offs and unexpected behaviors. Finally we draw conclusions that will inform the refinement of the design choices initially elaborated.

\section{BACKGROUND}

In this work, mood is considered as the measurable articulation of emotions and subjective feelings. The Circumplex Model of Affect [7] proposes that emotional concepts are organized according to a circular structure, in a 
two dimensional space of valence and arousal. Valence is a pleasure-displeasure space (often evaluated as "feeling good/positive" or "feeling bad/negative) while arousal indicates the level of energy or physiological activity (often described as having high or low energy) (Figure 2). Each emotion can be described as a linear combination of these two dimensions, or as varying degrees of both valence and arousal.

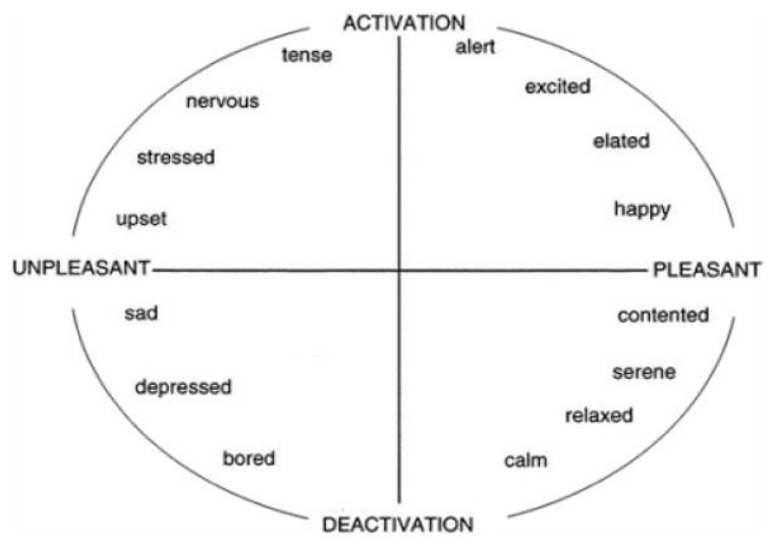

Figure 2: Circumplex Model of Affect (Russell, 1980)

Several studies related to the influence of emotions in daily activities have been carried out. Matic et al. [8] aim to monitor daily work routines of researchers and infer their influence on mood. They employ a mobile phone to monitor workers' routines and afterwards uncover correlations between these daily routines and mood states. Monitoring includes indoor localization with FM radio, sensor-based sound interaction and speech recognition, body movements with accelerometers and sleep quality deduced from self-rating questionnaires. The mood states are inferred through different methods of selfreporting based on questionnaires about subjective feelings. Morris et al. [9] investigated the potential of a mobile phone application to extend access to cognitive behavioral therapy techniques. This application is based on a two-dimensional representation of the circumplex model of emotion [7] which is generally called mood map. The intent of the application was twofold: emotional self-awareness by illuminating data trends and self-regulation through some interventions offered by the application. The application was deployed in a onemonth exploratory study with eight participants, aiming to compare the sampling data from the application with personal open-ended interviews about the use of the device and stressful incidents perceived by the participants.

The just reviewed projects have an individual perspective, offering users the possibility of being self-aware of their moods and correlate possible relationships to their activities. However, there are a number of projects that support mood sharing. For example, the MoodJam Project [10] provides a personalized visualization of moods and other people's moods based on user-defined colored strips and words, learning about trends and sharing results. The goal is to increase mood awareness among the users. Sundström et al. [11] developed a prototype called eMoto, a mobile service for sending affective messages to others. It supports the enrichment of messaging and communication with emotions, which are entered through a range of affective gestures and then mapped to a series of colors, shapes and animations shown in the background of a text message. The authors carried on a user study with five participants and five participants' friends as observers. The users were able to report and reflect on their communication patterns, their friendships, the experience of their own emotions and reactions to the emotional messages they received. Results show that emotional communication must prove ways to maintain the sometimes-fragile communication between friends, and not only support a simple transferring of information plus emotion.

MobiMood [12] is a social mobile application developed at Telefonica Research that enables groups of friends to explicitly share their moods with each other. They present an exploratory field study with the purpose of studying the impact on awareness and the role that explicit mood sharing has on communication. Additionally, they define a set of design implications for future social mobile awareness applications. Design implications include the importance of offering a full self-expression to the users; the need for highlighting context; the need for a social purpose, interaction and social visibility; as well as the enhancement of communication, identifying a communication channel to support. However, MobiMood does not aggregate or anonymize the information, publicly displaying the individual moods of the members of a friends group.

Despite allowing mood sharing, it must be noticed that these applications do not take into account the aggregation of mooddata. Moreover, anonymization plays a key role when systems offer the possibility of sharing such personal data like moods and therefore the intention and design of the application defines the level of privacy that the user must have, i.e. users' concerns are not the same when sharing their personal data with family, friends, work colleagues or strangers.

One remarkable approach in the field of behavior tracking with the purpose of improving oneself is the so-called lifelogging, pursued by a community known as Quantified Self [13]. Quantified Self is a collaboration of users and toolmakers who share an interest in self-knowledge through selftracking with the motto "self-knowledge through numbers". This interest results in a variety of tools to collect personally relevant information for self-reflection and self-monitoring, with the purpose of gaining self-knowledge about one's behaviors, habits and thoughts. Among these tools developed to track and visualize personal data generated by our own behavioral activities we can also find mood-related approaches. One example is Moodscope [14], a web-based application that allows users to measure their mood, track its evolution on a graph and share the scores with friends with the purpose of receiving support and help. Moods are measured using an online card game, and they are automatically shared by email. Another approach is a research project at the London 
School of Economics called Mappiness [15], whose aim is to know how people's happiness is affected by the local environment (pollution, noise, location, nearby people, etc.). Mappiness is based on an iPhone application which automatically measures location and noise-level and allows users to enter their mood based on three emotions (happy, relaxed and awake) and context information (e.g. what the user is doing or with whom).

Finally, the last tracking application reviewed is MoodyMe [16]. It allows users to log their mood, note what affects it, take pictures and relate them to a specific mood and record any treatments they are taking. Its interface is based on a calendar where the tracked moods of each day are represented by colored emoticons.

Although we reviewed some web-based application, the majority of the reviewed work has chosen to support mobile mood-tracking via smartphone applications. However, none of them was based on a combination of mobile and static interfaces in order to be tailored to different scenarios. Regarding the interfaces to represent emotions and moods, several representations have been found. Some of them are based on previous work made by psychologist, e.g. a mood map based on Russell's model or keywords based on Plutchik's model of eight primary emotion dimensions; while others are looking for the intuitiveness for the users, as it may happen with emoticons.

\section{DESIGN CHOICES}

Information systems to provide mood awareness have to undergo many design challenges due to the high variability of usage settings they must support; moreover they need to turn the qualitative nature of moods into a formalized representation that can be stored, analyzed and shared. We believe that to benefit from mood awareness a continuous support to the users must be provided; rather than working towards a one-fits-all solution we consider the use of pervasive interfaces where the user can choose according to her ongoing context.

Building on related works, we formalized the process of enabling mood awareness in a three-phase loop (Figure 3). Design criteria to inform the design of systems to enable mood awareness have been elaborated for each phase:

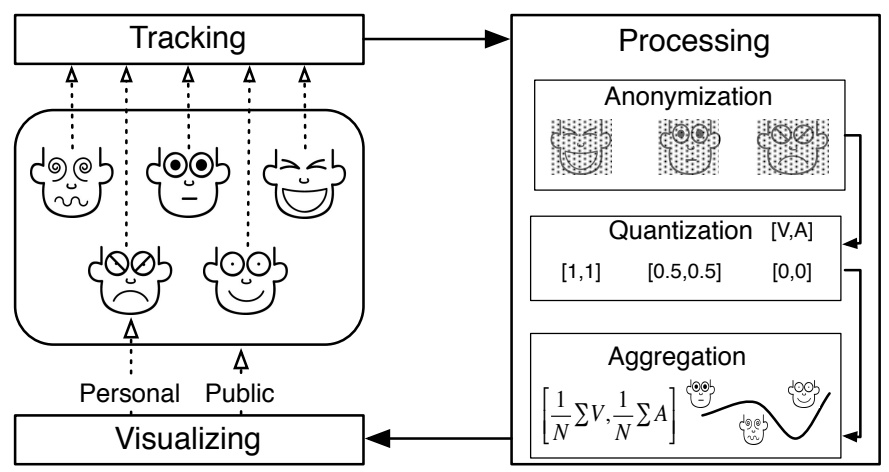

Figure 3: Process for supporting mood awareness. a. Tracking mood data

b. Processing mood information

c. Visualizing shared mood information

Mood tracking is enabled by desktop and mobile applications to allow sensing of mood information from multiple users (a). Information coming from different individuals is processed to be anonymous, quantified to a common scheme and get aggregated by means of statistical functions (e.g. average, variance). The aggregation outcome is shared (b). The users are made aware of the aggregated value via multiple visualizations (c), to be displayed via personal and public user interfaces.

A set of design choices has been elaborated with the aim of supporting the design of pervasive applications, providing multiple interaction modalities the users can choose according to an ongoing context. Each application may therefore implement a different degree or a subset of following design choices to address specific use cases in particular contexts.

The identified design choices are listed for each phase. The reader might notice that the design choices (DCs) for the tracking phases are mirrored by DCs in the visualization phase.

\section{A. Tracking}

\section{[DC1-T] Role of users}

Systems might be designed to make users active in the collection of data, passive, or a combination of the two characters. Systems that are designed for passive users collect mood data via tools such as sensors and algorithms that analyze human behaviors, e.g. facial expressions. Mood assessment is continuous in time, but data must be filtered in order to detect changes of mood. Moreover mood information must be filtered from data collected not related to the ongoing working activity (e.g. mood information during off-work time). Active users provide information about their moods via a computer interface (e.g. touchscreen, vocal input, gestures). Mood assessment is therefore discrete in time and the relevance of data is high, since it is likely that the users focus on providing feelings directly relates to an ongoing activity, hence avoiding external factors which might add information bias. Systems adopting an active/passive combined approach might rely on stress sensors or on scheduled events to detect changes of mood and proactively engage the user in providing actual mood information, e.g. asking for "How do you feel now?". To increase the level of engagement, users can be rewarded for their feedback (e.g. by showing information about peers' average mood). This rewarding may motivate users to regularly track information about themselves.

\section{[DC2-T] Degree of mobility}

Interfaces supporting mood tracking can be desktop, mobile or ambient-based. Desktop-based interfaces are suitable to be used in conventional meeting settings (e.g. roundtables) while mobile interfaces (e.g. smartphone-based) are effective to support mood tracking in spontaneous meetings or collaborative settings characterized by a high degree of 
mobility, e.g. an hospital ward, or where the access to traditional desktop interfaces is not possible. Ambient devices are able to support mood awareness without interrupting the user's activity; moreover users can interact with ambient devices employing less cognitive effort than traditional interfaces. A seamless integration of the three approaches: desktop, mobile and ambient, allows for continuous mood tracking.

\section{[DC3-T] Level of abstraction}

Whereas mood tracking is based on an active users approach (see DC1-T) user interfaces should represent mood information with a level of granularity adequate to the context of usage. Despite desktop interfaces can fully implement graphical, high-granular, representations based on the Circumplex Model of Affect [7], mobile and ambient interfaces should adopt metaphors based on that model. Fast, low-granular input interfaces based on emoticons or free form gestures [11] can be adopted.

\section{[DC4-T] Degree of intrusiveness}

Sensor-based mood tracking can be considered too invasive for some groups of users, and it can throw serious privacy concerns due to its perceived "always on" feature. On the other side, it does not require to interrupt the user to collecting mood information. User initiated mood inputs, can be perceived as more privacy-safe but users have to interrupt an ongoing activity to submit mood information to the system.

\section{[DC5-T] Degree of sharing}

Both personal (private) and shared moods are important to support reflection. Tracking private moods allows for reevaluating a past experience from an individual point of view. Sharing moods allows for evaluating personal feelings against the status of fellows co-participating in an activity; thus discovering discrepancies the users might be interested to reflect upon. Mechanisms for allowing choosing a level of sharing for each mood provided should be granted, e.g. anonymous, group-based and public.

\section{[DC6-T] Degree of contextualization}

Context information should be collected and stored with mood data in order to support the whole awareness process by giving clues on what has provoked a change of mood. Examples of context information are timestamps, textual and vocal notes, GPS locations, and photos. For example, matching the progression of moods with user-generated annotations can uncover correlations with events that have provoked change of moods. Moreover it allows for reflecting on moods on a temporal basis to unearth trends and peaks.

\section{B. Processing}

\section{[DC7-P] Degree of aggregation}

Extracting features such as statistical indexes (e.g. average, variance) from shared moods provides an aggregate overview of a workgroup mood in a determinate instant of time. It requires a degree of sharing (DC5-T) to be implemented by the tracking applications.

\section{[DC8-P] Degree of comparison}

Checking personal moods against an aggregate value to undercover similarities/differences can be helpful for reflection. Mechanisms for allowing comparing personal moods with a shared aggregated value to compute similarities and differences must be considered.

\section{Visualizing}

[DC9-V] Role of users

Mood awareness can be presented to users either with push or pull mechanisms. In the former case an aggregate mood value can be continuously given by public displays or via an embodiment, in the latter case the information can be provided on user's request, e.g. via a mobile application.

\section{[DC10-V] Degree of mobility}

As already highlighted in DC2-T, to support continuous mood awareness during mobile work, user-interface both for tracking and visualizing must implement some degree of mobility.

\section{[DC11-V] Level of abstraction}

As already pointed in DC3-T for the tracking phase, level of abstraction of the information visualized by computer interfaces should be set accordingly to the context of usage. Moreover, other than being a source of information, visualization must please the users being aesthetically coherent.

\section{[DC12-V] Degree of intrusiveness}

While allowing peripheral awareness of aggregated mood values, visualization interfaces should proactively notify the users whereas a change of mood that might trigger reflection happens. Information should migrate from the background to the foreground of the user's attention only when needed to trigger reflection.

\section{[DC13-V] Degree of sharing}

As part of a reflection process, shared moods can trigger conversation among participants in an activity. Applications should therefore implement social features in order to support and share conversation during and even after the mood-tracked activity.

\section{[DC14-V] Degree of contextualization}

Time-based visualizations of moods and relative context elements are useful to show correlations and trends in mood information collected during the tracking process (DC6-T).

The selection of a subset of design choices is scenariodriven. It is remarkable to observe that although the design choices are loosely coupled, they are not independent. Choosing a degree of implementation of a DC can affect or restrict the design space of another one (e.g. a high degree of mobility may limit the level of abstraction the application can 
provide). System designers should find the trade-off that fits best the scenario requirements ad hoc.

\section{ARCHITECTURE AND PROTOTYPES}

As part of the design study, we implemented the design choices (DCs) described in section III in a collection of prototypes, and we integrated them in a common architecture. Our prototypes are pieces of software running on retail hardware. One of the created visualization interfaces runs on a mesh-up of two devices. The aim is to evaluate in a real setting the user acceptance of the design choices and to highlight challenges. Some prototypes implement both tracking and processing phases DCs, while others are visualization interfaces and don't provide input mechanisms. Applications for the processing phase are remote services and they don't provide use interfaces.

The prototypes developed for each phase are presented in table 1:

TABLE 1

PROTOTYPES IMPLEMENTED

\begin{tabular}{|c||c|c|c|}
\hline PROTOTYPE & TRACKING & PROCESSING & VISUALIZING \\
\hline \hline MoodMap & $\bullet$ & & $\bullet$ \\
\hline MoodTimeline & $\bullet$ & & $\bullet$ \\
\hline MoodAvg & & $\bullet$ & \\
\hline MoodDisplay & & & $\bullet$ \\
\hline NabazMood & & & $\bullet$ \\
\hline TwittMood & & & \\
\hline
\end{tabular}

\section{A. Applications for tracking and visualizing}

\section{MoodMap App}

The MoodMap App (see Figure 4), engages the user in selecting a mood on a two-dimensional map that is based on Russel's Circumplex Model of Affect [7]. The MoodMap App allows users to introduce their mood and share it with other users [DC5-T]. Given that it is a web-based application, it is accessible from any web browser, i.e. from any device that provides a browser [DC2-T, DC10-V].

Regarding the interface, according to Russell's model, the horizontal axis of the map indicates valence and the vertical axis represents arousal. This diagram uses a colored background to indicate the different emotions that are related to the abstract valence and arousal values [DC3-T]. Both axis are numerically represented in the range $[0,1]$. The mood can be entered by clicking in the mood map (Figure 4a) and therefore mood capturing is made through a user-initiated mechanism [DC1-T].

After a mood-entry, the user gets rewarded with a graphical comparison of his own mood against the average mood [DC9V] (figure $4 b$ ). This reward builds on curiosity of the user. The visualization of the compared moods shows two colored bars, the first bar for valence and the second bar for arousal. This way, the user can easily compare both dimensions [DC8-P, DC11-V]. The vertical bars represent the range of values, numerically comprised between 0 (bottom) and 1 (top). The blue arrows show the values introduced by the user, whereas the black arrows show the average mood (Figure 4b). A "Back to Mood Map" button, placed at the bottom of the site, allows the user to go back to the mood map (initial state of the application) and introduce new mood values when desired.

(a)

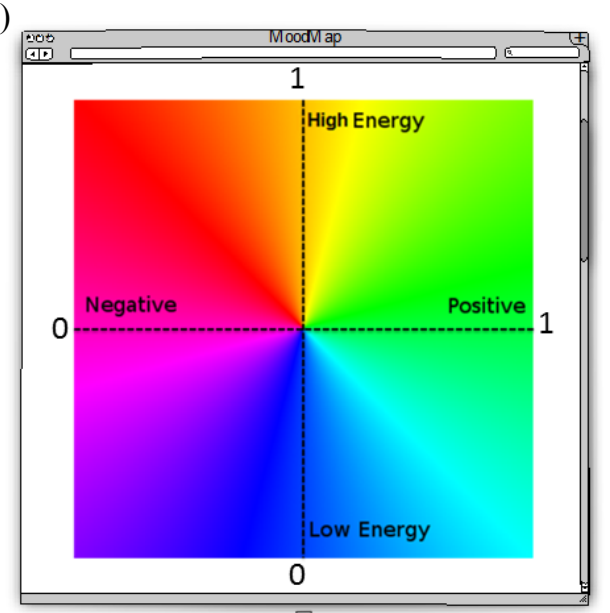

(b)
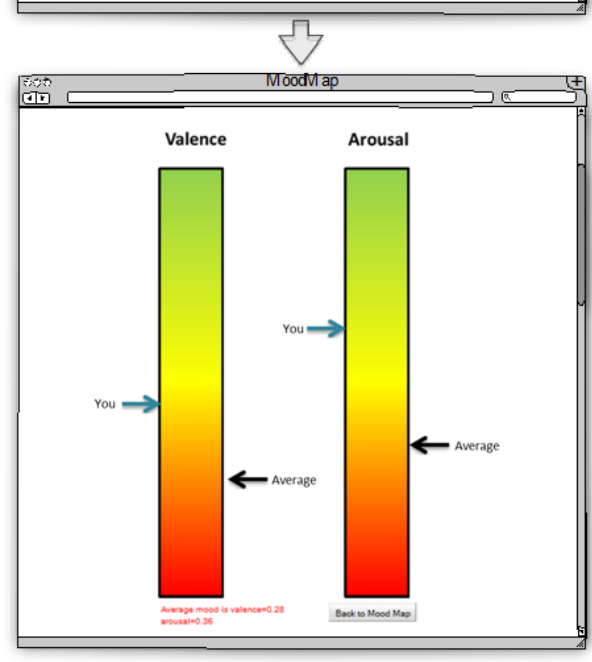

Figure 4: a) Bi-dimensional mood map to introduce the user's mood, b) Bars visualization to compare the moods.

Here, users are not just giving input for the system, but receiving a personalized response and being able to derive a benefit for themselves. This benefit of comparing themselves with the other users can lead them to regularly introduce their mood and therefore capture more data about themselves.

\section{MoodTimeline}

MoodTimeline is a mobile application for tracking and visualizing moods in a timeline together with contextual information (e.g. text, photos) [DC6-T]. It is part of a broader project aiming to collect and share experiences [17] and it runs on Android smartphones and tablet devices. Due to the limited dimensions of the display available on mobiles, it might be difficult to show moods on a map (as the one shown by MoodMap app). We have therefore decided for a low granular mood representation based on emoticons [DC3-T, DC11-V]. The mood map is divided into four quadrants, matching four different mood statuses, and we mapped those quadrants to four different emoticons that the user could choose from to track her mood (see table 2) 
TABLE 2

MOODMAP APP AND MOODTIMELINE MOOD ABSTRACTIONS MAPPING.

\begin{tabular}{|c|c|c|}
\hline Quadrant & $\mathrm{X}$ and $\mathrm{Y}$ values & Representation \\
\hline Top Right & $\begin{array}{l}\mathrm{x}(0,5-1,0), \\
\mathrm{y}(0,5-1.0)\end{array}$ & ( \\
\hline Top Left & $\begin{array}{l}\mathrm{x}(0,0-0,5), \\
\mathrm{y}(0,5-1.0)\end{array}$ & (2) - nervous \\
\hline Bottom Right & $\begin{array}{l}\mathrm{x}(0,5-1,0), \\
\mathrm{y}(0,0-0,5)\end{array}$ & ( - calm \\
\hline Bottom Left & $\begin{array}{l}\mathrm{x}(0,5-1,0), \\
\mathrm{y}(0,0-0,5)\end{array}$ & $0_{-\mathrm{sad}}$ \\
\hline
\end{tabular}

Users can add their mood to the timeline at any moment, by selecting one of the emoticons. The app supports attaching also other type of content to the timeline, e.g. audio, video, photos and comments (see Figure 5)

Adding media in a timeline provides a context for the moods submitted to the system. Reviewing contents on a temporal basis allows finding correlations among information (e.g. text notes, images, audio) and personal moods, which are beneficial for reflection [DC14-V]. The instant average mood is also visible to the user in a pop-up message [DC9-V].

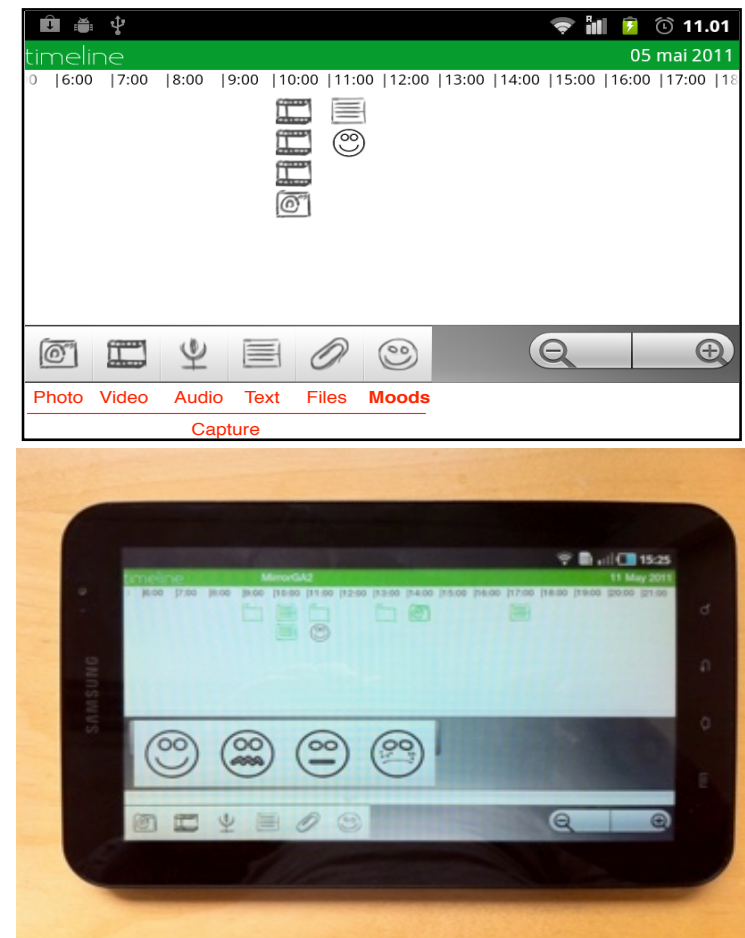

Figure 5: Several contents can be attached to the timeline, moods are among these.

\section{B. Applications for processing}

\section{MoodAvg}

Mood information provided by tracking applications are processed by a server in order to extract an aggregate value to be notified to the users. MoodAvg is a cloud-based application that calculates the average mood weighted [DC7-P] on the latest 10 mood-entries, provided by the tracking applications and devices. The mood-average is computed by averaging the valence and arousal components separately as in (1).

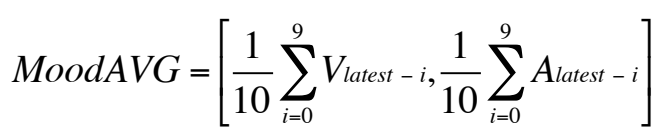

MoodAvg also computes the gap between personal moods and the average value [DC8-P] applications can show, for example see figure $4 \mathrm{~b}$.

\section{Applications for visualizing \\ MoodDisplay}

Large public displays are ambient devices which are useful to show information such as charts and images that can be seen from distance. We envision a possible use of this technology to visualize mood information during spontaneous meetings in corridors and halls. Our current prototype, MoodDisplay, shows the average mood on a mood map and the valence and arousal component via graphical gauge widgets [DC11-V] (figure 6). The visualization is updated every 15 seconds, only the latest value provided is shown [DC14-V]. This visualization, being "always on" [DC9-V] allows several people to meet in a shared space thus helping triggering collaborative reflection.

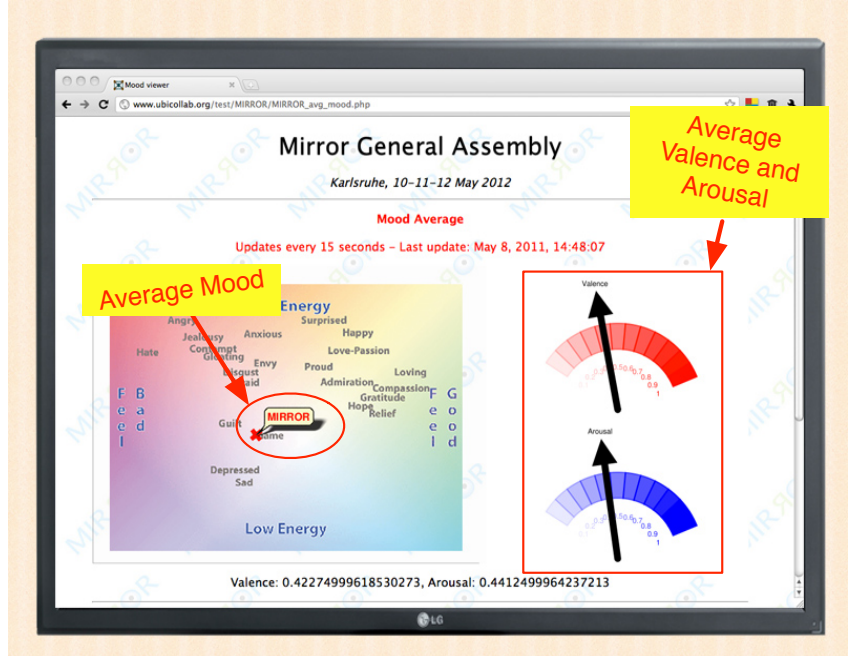

Figure 6: MoodDisplay, a public display to visualize the average mood.

\section{The Nabazmood}

The Nabazmood is a WiFi-enabled ambient device [DC10$\mathrm{V}]$ in a shape of a rabbit (Figure 7). The current prototype is a hardware mesh-up of a Nabaztag [18] and a pico-projector. Ambient devices are electronics characterized by their ability to be perceived at-a-glance, exploiting the capability of the human brain to perceive information without (or with low) cognitive load. Nabazmood is able to provide a visualization of average mood by separating the valence and arousal components and showing valence through ears movements and arousal through changing of colors [DC11-V]. 


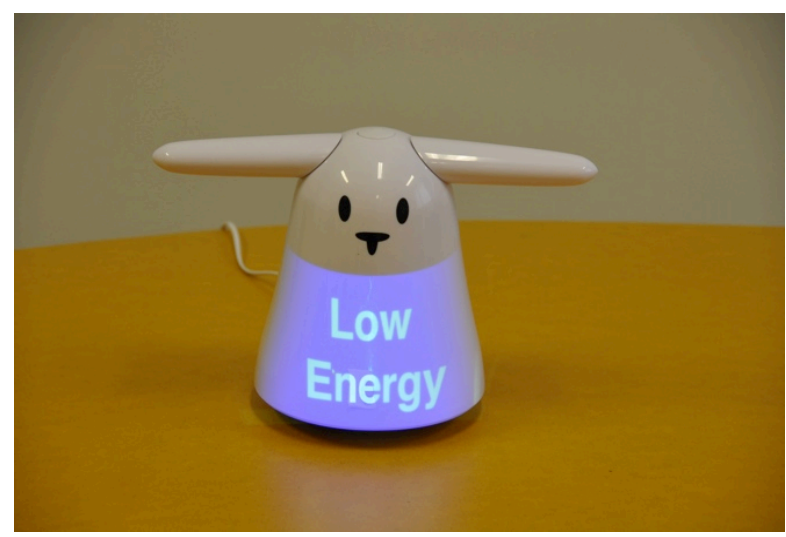

Figure 7: The Nabazmood, a moody rabbit.

For example, the rabbit pulling up its ears means that there is a high average level of valence, while changing color from red to blue means a lowering of arousal. Figure 8 shows some possible state the rabbit can assume and their related meanings in the mood map-domain.

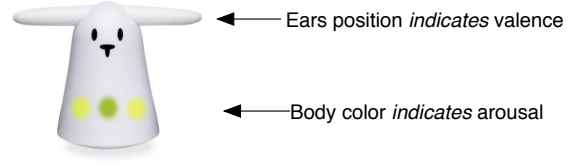

Examples:

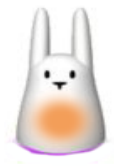

Happy

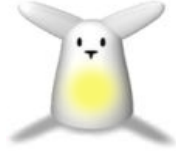

Afraid
Figure 8: Possible states of Nabaztag and meaning

Nabazmood provides an always-on non-disruptive assessment of average mood [DC9-V], of the latest mood aggregation [DC14-V]. Participants can monitor the rabbit behavior with a low cognitive effort, monitoring it subconsciously until a change in the rabbit behavior draws their attention.

\section{Twittmood}

The average mood is also twitted every 15 minutes. Our first prototype only shows valence and arousal values by float numbers ranging between 0 and 1 ( 0 minimum, 1 maximum) [DC11-V]. A more meaningful informational representation (for e.g. using feeling names) to be twitted is currently under investigation. A (non-human) twitter user has been created and it is possible for other twitter users to follow it [DC9-V] and to leave comments on average mood statuses [DC13-V] (Figure 9). Twitter also keeps storage of all the tweets generated by the system thus allowing reviewing past feelings [DC14-V]. Twitter is available for many platforms both desktop and mobile [DC10-V].

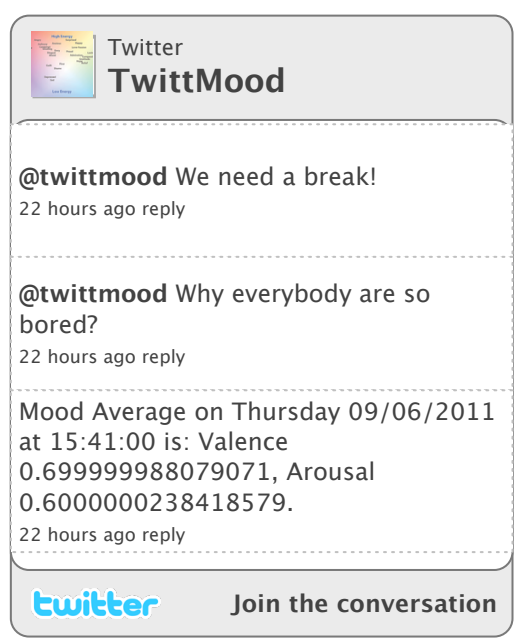

Figure 9: A shared mood average debated on twitter

\section{Architecture}

Figure 10 schematizes the overall architecture that integrated the above presented applications.

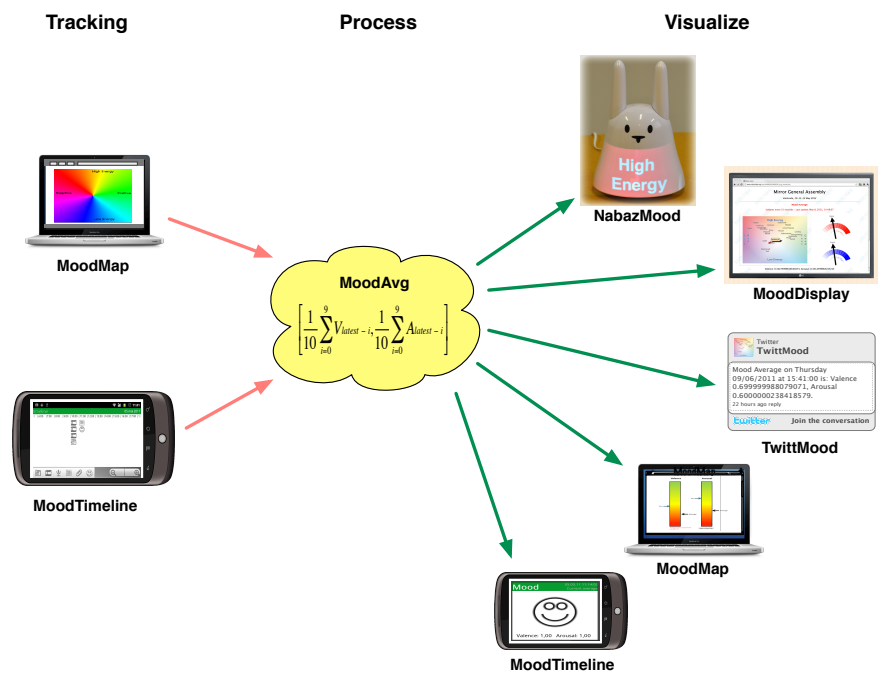

Figure 10: Overall architecture of the applications for acquisition and visualization of moods.

APIs for data exchange among applications have been developed in order to test the integration of different mood acquisition and visualization modality.

\section{Evaluation}

\section{A. Methodology}

All applications were evaluated in a three-day project meeting with 32 participants. The meeting days consisted of presentations and subsequent discussions in plenary and group sessions. Participants discussed several critical issues for the project and were focused on these aspects. This meeting was a promising test environment because (a) participants shared a common work context, (b) participants were in a real work situation and (c) the meeting was well structured and documented. 
At the beginning of the meeting, the experiment was shortly presented and additional mobile devices were given to volunteers. During the meeting, participants could state their mood with the different tools and see the changes in the average through the different visualizations.

Entered mood-data and the means of capturing were stored for later analysis. Additional data that was captured using the mobile devices was stored as well. The meeting agenda, the meeting protocol, additional notes and observations serve as a reference to analyze the captured data.

Additionally, participants were asked during breaks for feedback on their experience with the system and its usefulness for reflection.

\section{B. Captured Data and Observation}

During the three days, more than 1100 mood entries were captured. The meeting was observed to shed light on the relation between captured mood data and work context. Figure 11 shows the average arousal and valence during the first day of the meeting.

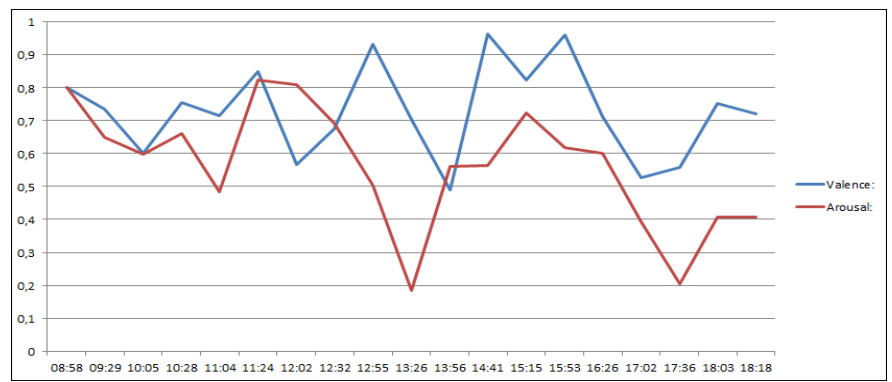

Figure 11: Average arousal and valence during the first day

The arousal line in Figure 11 shows how the arousal goes down in expectation of the lunch break and the end of the meeting. At these points, participants entered their mood to achieve a desired reaction of the presenter or the moderation, e.g. having a break, ending a discussion or closing the day. Afterwards, they pointed at the projected mood or complained over the delay of the displayed mood. Other trends could be identified too:

- When a heated discussion took place the average arousal increased.

- The valence decreased if the discussion was not evolving satisfactory.

- When the meeting was reaching the end of the last session the valence level decreased till reaching very low values ("sad").

Participants actively referred to the displayed average mood several times during the second day. Presenters started their presentations with sentences like "I hope I can keep this arousal level" or ended a presentation with sentences like "Look the Nabazmood, low arousal, we should have a break!" While these statements always had a mocking tone, the result was the desired break.

The captured data also shows that during the meeting the online web application was preferred. The mobile MoodTimeline was used mainly during breaks and during a lab visit in the afternoon. During the lab visit additionally pictures were taking using the mobile application.

On the second day, we observed some unexpected behavior. Single participants tried to manipulate the mood values by spamming the system with mood statements. They later explained that they wanted to see the Nabazmood to move its ears into a certain position.

\section{Feedbacks from Participants}

Informal feedbacks were collected by short interviews during breaks and by analyzing posted messages in the MoodTimeline. The results concern the idea to capture moods, the design of the apps and the relation between aggregated mood values and the meeting.

Statements on the mood capturing and deployed applications were in general very positive, especially the visualization using the Nabazmood rabbit was appreciated, since most discussions evolved around the two ambient visualizations.

When asked about the different user interfaces, participants highlighted the suitability of the different interfaces according to their mobility. They pointed out that a mobile application like the MoodTimeline was much more helpful and more used when they were mobile and that a web-based application like the Mood Map App was more suitable when attending the different presentations at the meeting room.

Participants could see relations between the aggregated mood data and the work process, in this case the meeting. For instance two participants sent the following two messages:

\section{"Does the discussion of the deliverables affect the mood of the} group?" and "For sure it did!".

One participant even considered to introduce an automatic alarm:

\section{"There should be an automatic break alarm when energy gets} below a specific level”.

Other participants tried to interpret the data:

"Low arousal in the group now. Do people need lunch? Good valence though :-)”.

Furthermore, participants discussed the displayed mood with each other and draw conclusions on the relation between current topics and reaction of the group. The MoodTimeline served as a source of tracking reference data too, i.e. information about what is happening and that can be directly connected to the available moods to offer richer context information. In this direction, users introduced comments like "Storyboards, finding the relationships with the user studies" or "Clustering cards... with suggested reflection activities".

The integration of two different abstractions for the mood input, the mood map and the emoticons, was evaluated positively. The users could understand it intuitively and appreciated the integration of different interfaces that allowed a richer collaborative mood collecting. 


\section{DISCUSSION}

The high amount of mood entries and the positive feedback of users indicate a high user acceptance. Users are willing to capture and share their mood. While we expected the playful user interface or the curiosity of the user to be most important, the possible influence on the meeting turned out to be the biggest motivator. The relation between average mood and the meeting is visible from the captured mood data and was confirmed by participant's feedback. However, our implementation of the mood average has several flaws that were exploited during the test.

The applications created awareness of the average mood during the meeting, however due to the user-centered nature of our mood tracking interfaces awareness is only made of moods of active users (see [DC1-T]) while we do not have any information from users who didn't send moods. We conclude that the system, besides keeping mood-entries anonymous, should highlight the level of involvement of users in building awareness and the distribution of moods-entries over the crowd; this can be considered as an index of plausibility of the awareness. Scenarios that require involvement of every user in mood awareness should rely on approaches where users have a passive role like sensor-based systems rather than an active user approach.

Providing different level of abstraction in input mechanism [DC3-T] (mood map and emoticons), although allowing tracking interfaces to be context-aware, it adds bias during the aggregation [DC7-P] phase. Fine-grained moods as those one provided on a 2-D space by the MoodMap App are aggregated with low-grained, discrete, moods provided by emoticons on the MoodTimeline. The trade-off between system accuracy and freedom, allowing the users to choose the interface that fit best an ongoing context, should be designed according to the usage scenario.

Besides meeting speakers actively reminded the crowd to share moods, users were not asked to submit moods on a regular-basis. Observations have shown that people engaged in heated discussion tend to not send mood entries meanwhile, leaving the aggregated mood to be influenced by users not part of the discussion

Observations have shown that some users manipulate the system by spamming mood-entries. Interviewed users admitted to behave in that manner as a way to play with the Nabazmood rabbit. A future implementation should only consider the last mood statement of a person. This would require user management techniques.

Two groups of users where identified:

- Users who referred to the system and discussed the average mood with others, thus supporting a collaborative reflection process; this is what our system was made for [DC6-T, DC13-V].

- Users who were interested in influencing meetings by "voting" with their mood entries, in order to support coordination of collaborative work.

While both groups contribute to provide mood awareness, this leads to the question, if the captured mood really reflects the average mood of the group.
Given the amount of data and the relation between meeting and average mood, the collected data could be used for later reflection. However, the evaluation was limited to three days in a prepared environment that visualized the average mood in different forms. Therefore it is unclear if participants would continue to use the application in other contexts.

\section{CONCLUSIONS AND FUTURE WORK}

We have presented a design study on mood awareness in collaborative environments attending to the design dimensions necessary to provide mood awareness and the influence of this awareness on the participants. We have seen that mood tracking provides an alternative channel for participants in a meeting to anonymously communicate their opinion and mood. Our results show that there is a strong relation between the context or activity taking place and the mood of the participants. Besides, we have presented several cases where mood awareness had an influence on the participants' behavior.

Our current approach aimed at minimizing the barriers for potential users. In result, the current system does not have a user management technique. A future solution should offer a user management to prevent mood spamming. New users could use the system for a number of requests without registration to keep the barriers low. If the user likes the system and wants to send more mood request the user has to register.

The analysis of $\operatorname{logs}$ and data was centered on the features of the different interfaces and the behavior of the participants. Future studies in this direction will involve the analysis of the captured data to deduce more information, e.g. which was the most interesting presentation or which topic was the most interesting for the audience. For this purpose, the combination of the captured data with other available data would be necessary.

We have presented a user-initiated approach, especially attending to unobtrusiveness and motivation of the users to track their moods. Future approaches may include systeminitiated communication, e.g. the system may react asking users to submit their moods in presence of high-variability in mood average and to provide support not just to face-to-face meeting but also to synchronous and asynchronous meetings over distance.

\section{ACKNOWLEDGMENT}

We wish to thank Anders Kristiansen and Andreas Storlien for their precious help setting up the evaluation experiment and for giving us access to the data they collected.

\section{REFERENCES}

[1] J. Dewey, Experience and education. Kappa Delta Pi, 1938.

[2] P. Dourish and V. Bellotti, "Awareness and coordination in shared workspaces," Proceedings of the 1992 ACM conference on Computer-supported cooperative work, pp. 107-114, 1992.

[3] J. Bardram and T. Hansen, "Context-Based Workplace Awareness," Computer Supported Cooperative Work (CSCW), vol. 19, no. 2, pp. 105-138, 2010.

[4] S. Greenberg and G. McEwan, "Supporting social worlds with the community bar," Proceedings of the 2005 international ACM 
SIGGROUP conference on Supporting group work, 2005.

[5] D. Boud, R. Keogh, and D. Walker, Eds. Reflection: Turning Experience into Learning, 1st ed. Routledge, 1985, p. 170.

[6] M. Weiser and J. Brown, "Designing calm technology," PowerGrid Journal, vol. 1, no. 1, pp. 75-85, 1996.

[7] J. Russell, "A circumplex model of affect.," Journal of personality and social psychology, pp. 1161-1178, 1980

[8] A. Matic, A. Papliatseyeu, S. Gabrielli, and V. Osmani, "Happy or Moody? Why so?," 5th UbiHealth Workshop in conjunction with UBICOMP-2010. Copenhagen, Denmark.

[9] M. E. Morris et al., "Mobile therapy: case study evaluations of a cell phone application for emotional self-awareness.," Journal of medical Internet research, vol. 12, no. 2, p. e10, 2010.

[10] "MoodJam project," http://www.moodjam.org/.

[11] P. Sundström and A. Ståhl, "In situ informants exploring an emotional mobile messaging system in their everyday practice," International Journal of Human-Computer Interaction, 2007.

[12] K. Church, E. Hoggan, and N. Oliver, "A study of mobile mood awareness and communication through MobiMood," in NordiCHI '10: Proceedings of the 6th Nordic Conference on Human-Computer Interaction: Extending Boundaries, 2010.

[13] "The Quantified Self, self-knowledge through numbers," http://quantifiedself.com.

[14] "Moodscope - Lift your mood with a little help from your friends," http://www.moodscope.com.

[15] "Mappiness: maps happiness across space in the UK," http://www.mappiness.org.uk/.

[16] "Moody Me - Mood Diary App," http://www.medhelp.org/land/mood-diary-app.

[17] A. Kristiansen and A. Storlien, Enhancing reflection by collaboratively capturing experiences in a timeline, Norwegian University of Science and Technology Master Thesis, 2011.

[18] "Nabaztag," www.nabaztag.com. 